\title{
Autonomic testing of women with interstitial cystitis/bladder pain syndrome
}

\author{
Gisela Chelimsky $\cdot$ N. Patrick McCabe • \\ Jeffrey Janata $\cdot$ Robert Elston $\cdot$ Lu Zhang • \\ Sarah Ialacci · Thomas Chelimsky
}

Received: 4 February 2014/ Accepted: 26 March 2014/Published online: 30 April 2014

(C) The Author(s) 2014. This article is published with open access at Springerlink.com

\begin{abstract}
Purpose Interstitial cystitis/bladder pain syndrome (IC/ BPS) is characterized by urinary urgency, frequency, nocturia, pain worse as the bladder fills and improved after emptying. These features might suggest abnormal autonomic bladder control mechanisms. We compared the structural integrity of the autonomic nervous system (ANS) in IC/BPS and control subjects.

Methods IRB-approved study at University Hospitals Case Medical Center, Cleveland, $\mathrm{OH}$ to evaluate the structural integrity of the ANS in adult females. Testing included cardiovascular response to deep breathing, Valsalva maneuver, $30 \mathrm{~min}$ head up tilt, and sudomotor test. Results Differences in ANS integrity for IC/BPS subjects and controls were determined by modified Composite Autonomic Severity Score (CASS) that includes sudomotor, adrenergic and cardiovascular indices. Baseline heart rate (HR) and HRs from each of three $10 \mathrm{~min}$ upright segments of a tilt test were compared and trend analyses performed using $t$ tests. Healthy and IC/BPS subjects were demographically similar. The two groups did not differ in modified-CASS scores but elevated average peak heart rate was evident during baseline (supine; $p=0.057$ ) for IC/
\end{abstract}

G. Chelimsky · T. Chelimsky

Medical College of Wisconsin, 8701 W Watertown Plank Road, Milwaukee, WI 53226, USA

N. P. McCabe $\cdot$ J. Janata $\cdot$ R. Elston $\cdot$ L. Zhang $\cdot$ S. Ialacci Case Western Reserve University, 10900 Euclid Avenue, Cleveland, OH 44106, USA

T. Chelimsky $(\square)$

Department of Neurology, Medical College of Wisconsin, 9200 W. Wisconsin Ave., Milwaukee, WI 53226, USA

e-mail: tchelimsky@mcw.edu
BPS subjects prior to a tilt test. Difference at baseline was maintained at each interval during the tilt, with nearly identical slopes across intervals. The preliminary nature of this report denotes a small sample size and important differences may not be detected.

Conclusions The findings show no structural ANS abnormalities in IC/BPS subjects. Higher baseline HR supports the concept of functional rather than structural change in the ANS, such as abnormality of sympathetic/ parasympathetic balance that will require further evaluation.

Keywords Interstitial cystitis/bladder pain syndrome . Pelvic pain $\cdot$ Autonomic nervous system

\section{Introduction}

Interstitial cystitis/bladder pain syndrome (IC/BPS) classically comprises urinary urgency, frequency, nocturia, and chronic pain in the pelvis worse as the bladder fills and better once empty. Such clinical features might suggest disordered bladder control from a generalized autonomic nervous system (ANS) abnormality. This concept might explain the IC/BPS known comorbid conditions [5] such as irritable bowel syndrome, migraine headache and fibromyalgia, also associated with ANS function changes $[2,6$, $10,13]$. We hypothesized that IC/BPS belongs to a larger family of (often co-morbid) disorders that share common autonomic and sensory abnormalities. This study aimed to assess the structural integrity of the ANS in subjects with IC/BPS compared to healthy age matched controls. Based on abnormalities reported in co-morbid disorders such as fibromyalgia and migraine, we expected to find changes in responses to sudomotor and tilt table testing, with preserved 
cardiovascular responses to deep breathing and to the Valsalva maneuver. In addition, given the changes in heart rate variability found by others in chronic pain states, we thought it important to evaluate the structure of the ANS as a basis for drawing any conclusions regarding its structure.

\section{Participants and methods}

\section{Participants}

This prospective IRB-approved study (University Hospitals Case Medical Center, Cleveland, $\mathrm{OH}$ ) conducted in accordance with the ethical standards laid down in the 1964 Declaration of Helsinki and its later amendments evaluated the structural integrity of the ANS of adult females with diagnosis of IC/BPS and matched female healthy controls, as a portion of the larger ICEPAC (Interstitial Cystitis-Elucidation of Psychophysiologic and Autonomic Characteristics) study (ClinicalTrials.gov Identifier: NCT01616992). Data presented herein are from women enrolled in ICEPAC between 02/2011 and 02/2012 recruited through University Hospitals Case Medical Center (Cleveland, $\mathrm{OH}$ ), and Case Western Reserve University (Cleveland, OH), Summa Health System (Akron, $\mathrm{OH}$ ), and ResearchMatch.org. All subjects gave their informed consent prior to inclusion in the study. Study enrollment continues through June of 2014 with a goal of 38 healthy and 76 IC/BPS participants. Other ICEPAC subject groups are not presented here.

\section{Inclusion/exclusion criteria}

Subjects with IC/BPS required $\geq 6$ months of symptoms with pain clearly linked to bladder fullness, and exclusion criteria aligned with the IC/BPS-NIDDK criteria [1]. Healthy controls consisted of matched females, screened to exclude the presence of both IC/BPS and any manifestation (in the last 5 years) of any disorder commonly co-morbid with IC/BPS, including fibromyalgia by history or exam, chronic fatigue syndrome or unexplained fatigue for $>6$ months, chronic pain disorder of any type, complex regional pain syndrome, cyclic vomiting syndrome, irritable bowel syndrome, functional abdominal pain, functional dyspepsia, migraine headache, postural tachycardia syndrome, Raynaud's syndrome, reflex syncope, temporomandibular joint disorder, post-traumatic stress disorder, panic disorder, periodic limb movements in sleep, and multiple chemical sensitivity. Exclusion criteria for all subjects (healthy and IC/BPS) included pregnancy, breastfeeding, hematuria or infection on urinalysis, three urinary tract infections within the previous 12 months, pelvic or bladder neoplasm or infection, unstable medical
Table 1 Modified Composite Autonomic Severity Score (CASS)

\begin{tabular}{|c|c|}
\hline Sudomotor index & $\begin{array}{l}\text { Single QSART site reduced or distal sweat } \\
\text { volume }<1 / 3 \text { of proximal value } \\
\text { Single QSART site }<50 \% \text { of lower limit } \\
\text { Two or more QSART sites }<50 \% \text { of limit }\end{array}$ \\
\hline Adrenergic index & $\begin{array}{l}\text { Phase } \mathrm{II}_{\mathrm{E}} \text { reduction }<40>25 \mathrm{mmHg} \mathrm{MBP} \text {, or } \\
\text { reduced phase } \mathrm{II}_{\mathrm{L}} \text {, or pulse pressure reduction } \\
\text { to } \leq 50 \% \text { of baseline } \\
\text { Increased PRT time }(4-5 \mathrm{~s}) \\
\text { Phase } \mathrm{II}_{\mathrm{L}} \text { absent or increased PRT }(6-9 \mathrm{~s}) \\
\text { Absent phase IV } \\
\text { Absent phase } \mathrm{II}_{\mathrm{L}} \text { and } \mathrm{IV} \text { and increased PRT } \\
\geq 10 \mathrm{~s} \\
\text { The prior one plus orthostatic hypotension } \\
\text { defined as } \mathrm{SBP} \text { reduction } \geq 30 \mathrm{mmHg} \text {;BP } \\
\geq 20 \mathrm{mmHg}\end{array}$ \\
\hline $\begin{array}{l}\text { Cardio vascular } \\
\text { HR index }\end{array}$ & $\begin{array}{l}\mathrm{HR}_{\mathrm{DB}} \text { or } \mathrm{VR} \text { reduced but }>50 \% \text { of minimum } \\
\mathrm{HR}_{\mathrm{DB}} \text { or } \mathrm{VR} \text { reduced but }<50 \% \text { of minimum } \\
\mathrm{HR}_{\mathrm{DB}} \text { and } \mathrm{VR} \text { reduced but }<50 \% \text { of minimum }\end{array}$ \\
\hline
\end{tabular}

Each subsection allows for 1 score point (i.e. a total of 3 for sudomotor, 4 for adrenergic and 3 for cardiovascular HR index)

$Q S A R T$ quantitative sudomotor axon reflex, $M B P$ mean blood pressure, $P R T$ pressure recovery time, $S B P$ systolic blood pressure, $H R_{D B}$ heart rate response to deep breathing, $V R$ valsalva ratio

or psychiatric illness (such as untreated depression, psychosis, etc.), central or peripheral nervous system disorder (Diabetic Neuropathy-regardless of A1c level, Parkinson's disease, Alzheimer's, MS, stroke, etc.), inability to stop autonomically active or pro-kinetic (gastrointestinal motility modifying) agents prior to testing, current consumption of $>10$ alcoholic beverages per week, or any major surgical intervention in the last 90 days.

\section{Autonomic testing}

All subjects underwent ANS testing described in detail elsewhere [3] including the cardiovascular responses to deep breathing (DB, primarily testing cardiac parasympathetic integrity), the Valsalva maneuver (VM, testing cardiac sympathetic, parasympathetic and vasomotor sympathetic integrity), tilt table test (testing cardiac and vasomotor sympathetic integrity) and quantitative sudomotor axon reflex test (QSART, testing post-ganglionic sympathetic cholinergic integrity). The tilt table test was performed at $70^{\circ}$ for $30 \mathrm{~min}$. The VM (using $15 \mathrm{~s}$ and $40 \mathrm{mmHg}$ ) and DB were performed 3 and 2 times, respectively, in each subject.

Evaluation and statistical analyses

To quantify the results of testing, we utilized a modified Composite Autonomic Severity Score (CASS) [11] 
(Table 1), with sudomotor, adrenergic and cardiovascular heart rate indices. Some degree of anxiety may artificially inflate initial baseline pressures and heart rates, producing a false impression of an orthostatic drop. The tilt table test baseline was therefore taken from the last $3 \mathrm{~min}$ of the 5-10 min segment after return to the supine position, except if tilt table test was terminated due to a nearsyncopal episode, when a strong vagal surge may produce a non-typical post-tilt baseline, and the baseline was taken from the final $3 \mathrm{~min}$ of the first $10 \mathrm{~min}$ supine period prior to upright tilt. The upright portion of the tilt was divided in three 10-min segments with the mean of the three highest heart rates taken as the peak for each segment. Activity and symptom severity were evaluated every minute during the tilt table test, and outliers accompanying fidgeting and talking were excluded. Subjects unable to complete the first two intervals of the tilt table test (e.g. due to a syncopal episode) were excluded from HR analysis.

Data Analysis asked several questions: (1) Do IC/BPS and healthy control HR differ at baseline or when upright? (2) Is there an upright trend? (3) If a trend is found, is it linear or quadratic and is there a between group difference in that trend? Each of these questions was answered by calculating a linear contrast of the observed average peak HR for each of the subjects in the sample and performing a $t$ test. Since the average peak HR values and log-transformed values produced similar results, only the average peak HR values are shown. Age, BMI and modified CASS score between groups were also compared by $t$ test.

\section{Results}

This preliminary report includes 15 healthy women [mean age 36.3 years $\pm 16.1(\mathrm{SD})$; mean BMI $26.7 \pm 8.6]$ and 14 women with IC/BPS (mean age 36.5 years \pm 16.1 ; mean BMI $29.0 \pm 7.7)$. There was no significant difference in age $(p=0.98)$ or BMI $(p=0.46)$ between healthy and IC/BPS subjects. The analysis included two subjects with IC/BPS that were unable to stop medications (one was on diphenhydramine and the other on amitriptyline). The subject on amitriptyline had a modified CASS score of 3, with 2 points due to decreased sweat in the foot and 1 point for mild adrenergic dysfunction during the Valsalva maneuver, with no syncope and HR increased during the tilt of $<10 \mathrm{bpm}$ while upright. The subject on diphenhydramine had a modified CASS of 3 due to significant decrease in sweat output in the forearm and foot, with normal adrenergic and cardiovascular HR index, and HR increased by $33 \mathrm{bpm}$ while upright prior to a pre-syncopal
Table 2 Summary of autonomic testing findings

\begin{tabular}{llll}
\hline & IC/BPS & Healthy & $p$ \\
\hline Number of subjects & 14 & 15 & \\
Subjects with syncope & 2 & 1 & \\
$\begin{array}{l}\text { Subjects with orthostatic } \\
\quad \text { hypotension }\end{array}$ & 0 & 0 & \\
Sudomotor index & $1.8 \pm 1.3$ & $1.4 \pm 1.0$ & $>0.05$ \\
Adrenergic index & $0.6 \pm 0.5$ & $0.5 \pm 0.5$ & $>0.05$ \\
CV HR index & $0.2 \pm 0.4$ & $0.1 \pm 0.4$ & $>0.05$ \\
Modified CASS & $2.4 \pm 1.4$ & $2.0 \pm 1.1$ & $>0.05$ \\
\hline
\end{tabular}

Values are mean $\pm \mathrm{SD}$

episode. As the results from these two subjects did not differ significantly from the other subjects, they were included for analysis. No significant difference was observed between women with IC/BPS and healthy controls for any ANS integrity test as indicated by total modified CASS scores (Table 2). Three subjects with syncopal episodes in the first 20 min were excluded from the tilt table test analysis.

\section{Cardiovascular response}

Cardiac response to deep breathing was abnormal in three IC/BPS and in one healthy control. One healthy subject and one IC/BPS had a mildly abnormal Valsalva HR ratio. The cardiovascular HR index and the adrenergic index were similar in both groups (Table 2).

\section{Tilt table test}

All subjects underwent a tilt table test. Two IC/BPS subjects failed to complete interval 3; one fainted $1 \mathrm{~min}$ into interval 3 and one complained of discomfort $4 \mathrm{~min}$ into interval 3 and the tilt was discontinued. Two subjects in the healthy group and 3 in the IC/BPS had pre-syncope. Orthostatic hypotension did not occur. A difference in baseline HR (supine) between the groups approached significance $(p=0.057)$ and there is a significant linear trend after baseline, with no significant non-linear trend or trend by group interaction (Table 3; Fig. 1). Log transformation produced similar results with a slightly lesser fit (not shown). The $t$ tests are not independent but the correlation between contrasts for baseline and slope were not significant.

Quantitative sudomotor axon reflex test (Qsart)

Subjects in the healthy control and in the IC/BPS groups showed mild abnormalities of sudomotor function, with comparable modified sudomotor CASS scores (Table 2). 
Table 3 Summarized data from the tilt table test

Peak HR data presented as mean $\pm \mathrm{SD}$

${ }^{a}$ IC/BPS minus healthy control

b Data were pooled (healthy control and IC/BPS) as there was no difference between the fitted lines for each group. This created a model for all data post baseline

\begin{tabular}{llllll}
\hline & IC/BPS & Healthy & Estimate & $p$ & $95 \%$ CI \\
\hline $\begin{array}{l}\text { Number of subjects } \\
\text { Baseline }\end{array}$ & 12 & 13 & & & \\
$\quad$ Peak HR & $72.17 \pm 15.44$ & $62.08 \pm 7.48$ & & & \\
$\quad$ Group difference & & & 10.09 & 0.057 & $(-0.352,20.53)$ \\
Post baseline & & & & \\
Peak HR interval 1 & $90.50 \pm 20.83$ & $79.85 \pm 13.28$ & & 0.148 & \\
$\begin{array}{l}\text { Peak HR interval 2 } \\
\text { Peak HR interval 3 }\end{array}$ & $95.67 \pm 24.08$ & $81.38 \pm 13.04$ & & 0.086 & \\
$\begin{array}{l}\text { Group difference } \\
\text { Linear slope (pooled) }\end{array}$ & $98.58 \pm 25.02$ & $85.54 \pm 13.88$ & & 0.104 & \\
$\begin{array}{l}\text { Linear slope by group } \\
\text { interaction (non-pooled) }\end{array}$ & & & 12.99 & 0.106 & $(-3.050,29.038)$ \\
$\begin{array}{l}\text { Quadratic curvature } \\
\text { (pooled) }\end{array}$ & & & 3.16 & $<0.0001$ & $(1.876,4.445)$ \\
$\begin{array}{l}\text { Quadratic curvature by } \\
\text { group interaction (non- } \\
\text { pooled) }\end{array}$ & & & 1.70 & 0.186 & $(-0.887,4.278)$ \\
\hline
\end{tabular}

A

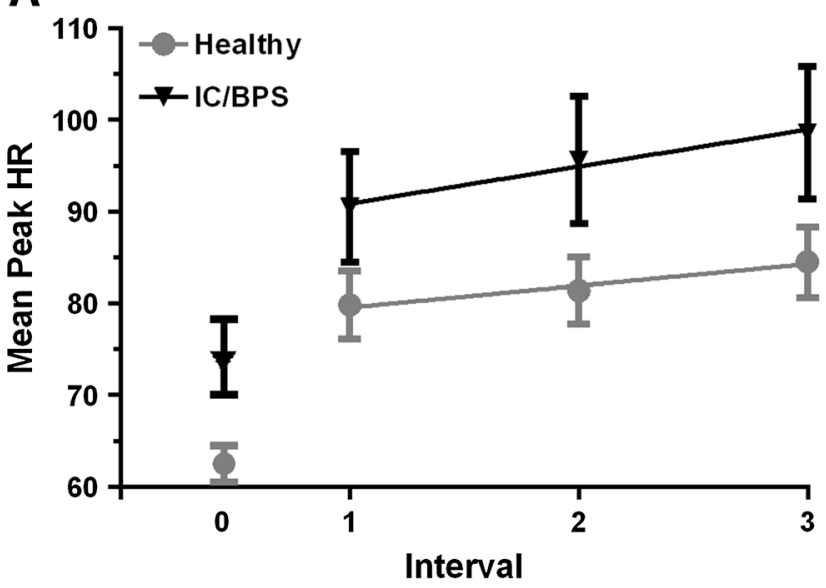

Fig. 1 Mean peak heart rate data at baseline (interval 0) and during the first, second, and third 10-min blocks (intervals 1, 2, and 3, respectively) of the upright tilt. a Slopes of post-baseline trends for

\section{Discussion}

To our knowledge, this is the first study to assess ANS integrity in patients with IC/BPS. While these data are preliminary, the aim of this manuscript was to evaluate the data accumulated to date and generate hypotheses that could be tested in the complete dataset. The ICEPAC study was designed to test the hypothesis that ANS structure may be abnormal in IC/BPS. Our data to date suggest that the null hypothesis prevails, i.e. that the structure of the ANS, at least as it innervates cardiovascular and sudomotor endorgans, is normal as evaluated by standard clinical autonomic testing.
B

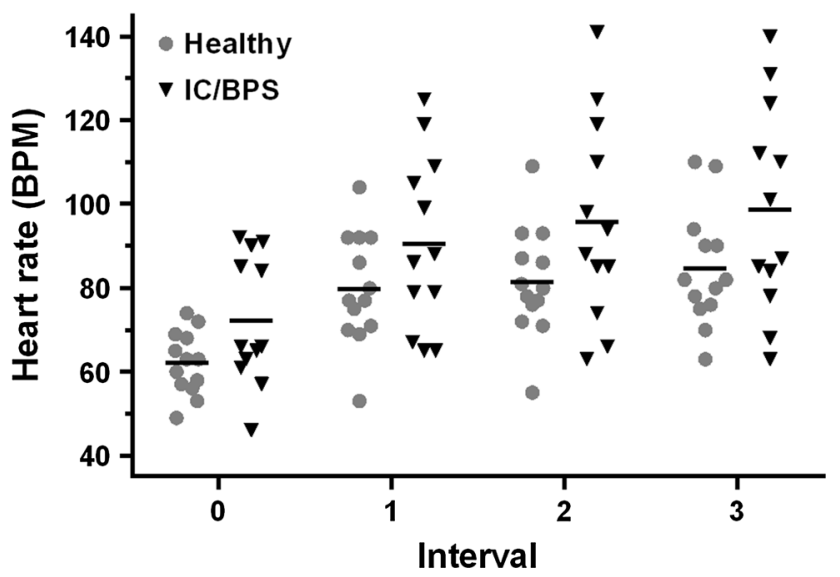

healthy controls and subjects with IC/BPS. Data are mean peak heart rate \pm SE. b Raw mean peak HR data points for each group at their respective intervals

To date, several studies have assessed various aspects of the ANS in a variety of painful disorders, for example heart rate variability in fibromyalgia [15] or irritable bowel syndrome [2] or heart rate and blood pressure change to bladder filling in IC/BPS [16]. However, none of these studies had undertaken the critical preliminary step of determining whether the structure of the ANS is intact. Without this information, one could not determine whether findings of abnormal ANS activity might be due to a structural change in the ANS, or to a functional abnormality associated with the disorder being studied. The current findings indicate that ANS structure in these patients does not differ from that of healthy matched 
females at least in IC/BPS, suggesting that observed ANS differences more likely relate to functional, not structural abnormalities. The observed increase in the baseline heart rate, as well as a trend for increased heart rate after $10 \mathrm{~min}$ upright, support a tentative conclusion of an abnormality in ANS responsiveness and function, rather than any structural change in the autonomic fibers or connections themselves. This hypothesis will be further explored in separate studies focused on ANS function, using heart rate variability analysis.

It is important to note that our healthy control group, despite being highly selected by history (previous 5 years) and detailed examination to confirm the absence of any signs or symptoms suggestive of a chronic pain, autonomic or neurologic disorder, did demonstrate some mild abnormalities. Such findings challenge our understanding of "normal" [4]. Moreover, these "abnormal" values may have obscured differences between the groups. Alternatively, the absence of a difference may simply represent a type II error-a larger cohort would demonstrate a difference. The subjects with IC/BPS that could not stop medication may have augmented abnormalities associated with the tilt test and autonomic testing due to anticholinergic properties of diphenhydramine and amitriptyline. However, these medications should have increased, not decreased, the difference between the groups [7, 9].

Studies of the ANS in patients with IC/BPS are scarce. One recent study found that hydrodistention of the urinary bladder induced a much greater heart rate, systolic and diastolic blood pressure rise in subjects with and without cystoscopic findings of ulcers and/or submucosal petechial hemorrhages [16]. The authors mentioned several possible interpretations, including (1) increased (although not reported) pain sensation in patients with ulcers; (2) different stages of the same disease or (3) two different diseases. Subjects with IC/BPS also have been shown to have increased baseline heart rate at rest when compared to controls, as well as some increase in the resting diastolic, but not the systolic, blood pressure [12]. We have found similar results, with higher baseline heart rate in the $\mathrm{IC} /$ BPS patients. However, in none of these studies had the structure of the ANS also been evaluated. The findings from our study lend credence to the idea that one can reliably examine ANS function in these patients. The increase in heart rate may be related to several factors: (1) amount of physical activity subjects are performing; (2) a functional rather than a structural change in the ANS such as an abnormality of sympathetic/parasympathetic balance suggested by our finding and that of others [12, 16], leading to a higher baseline heart rate; (3) higher anxiety level. The findings in this hypothesis generating study will allow further exploration of these possibilities.

This is not the first study to report a change in autonomic function in patients with a chronic pain disorder. A relative skew toward sympathetic influence also occurs in other functional pain disorders such as irritable bowel syndrome, fibromyalgia and chronic fatigue [2, 8, 14]. To date, no study has examined the meaning of these findings in the context of pain generating mechanisms to understand whether increased heart rate, for example, reflects a contributor or a consequence of a chronic pain syndrome. A prospective study will clarify the role of the ANS in chronic pelvic pain, and its relationship to psychological changes that occur frequently in these disorders. Future directions might include an assessment of heart rate variability at rest and after a physiologic or psychological stress or to extend the findings reported by Lutgendorf et al. [12].

In conclusion, although this study was performed in a relatively small number of subjects $(n=29)$, which could lead to a type II error (not finding a difference when one exists) it nonetheless constitutes the largest study of the ANS in IC/BPS to date. These findings require confirmation with a larger cohort, but suggest that ANS structure is not different in subjects with IC/BPS when compared to highly screened healthy subjects. Particular note should be made that this detailed screening of normal subjects required healthy subjects to have no disorders of any kind remotely known to harbor an association with any autonomic abnormality. Nonetheless, occasional mild abnormalities were present.

Acknowledgments The ICEPAC Study is funded by the National Institute of Diabetes and Digestive and Kidney Diseases (NIDDK) (R01DK083538). The following individuals are members of the ICEPAC Study Advisory Board and have helped shape the design and methodologies describe herein: Debra Erickson, M.D. (Dept. of Surgery, University of Kentucky College of Medicine, Lexington, KY, USA), Kathleen Pajer, M.D., M.P.H. (IWK Health Centre, Dalhousie University, Halifax, Nova Scotia, Canada), Julian Thayer, Ph.D. (Dept. of Psychology, The Ohio State University, Columbus, OH, USA), Ursula Wesselmann, M.D., Ph.D. (Dept. of Anesthesiology, UAB School of Medicine, Birmingham, AL, USA), Phyllis Zee, M.D. (Center for Sleep \& Circadian Biology, Northwestern University, Evanston, IL, USA), and Denniz Zolnoun, M.D., M.P.H. (Dept. of Obstetrics and Gynecology, UNC School of Medicine, Chapel Hill, NC, USA).

Conflict of interest The authors declare they have no conflict of interest.

Open Access This article is distributed under the terms of the Creative Commons Attribution License which permits any use, distribution, and reproduction in any medium, provided the original author(s) and the source are credited.

\section{References}

1. NIDDK (2006) 2006 NIDDK International Symposium: Frontiers in painful bladder syndrome and interstitial cystitis. Bethesda Marriott, Bethesda, MD 
2. Chalaye P, Goffaux P, Bourgault P, Lafrenaye S, Devroede G, Watier A, Marchand S (2012) Comparing pain modulation and autonomic responses in fibromyalgia and irritable bowel syndrome patients. Clin J Pain 28:519-526

3. Chelimsky G, Boyle JT, Tusing L, Chelimsky TC (2001) Autonomic abnormalities in children with functional abdominal pain: coincidence or etiology? J Pediatr Gastroenterol Nutr 33:47-53

4. Chelimsky G, Ialacci S, Chelimsky TC (2013) Autonomic testing in healthy subjects: preliminary observations. Clin Auton Res 23:113-116

5. Clemens JQ, Elliott MN, Suttorp M, Berry SH (2012) Temporal ordering of interstitial cystitis/bladder pain syndrome and nonbladder conditions. Urology 80:1227-1231

6. da Ribeiro RPC, Roschel H, Artioli GG, Dassouki T, Perandini LA, Calich AL, de Sa Pinto AL, Lima FR, Bonfa E, Gualano B (2011) Cardiac autonomic impairment and chronotropic incompetence in fibromyalgia. Arthritis Res Ther 13:R190

7. Freysoldt A, Fleckenstein J, Lang PM, Irnich D, Grafe P, Carr RW (2009) Low concentrations of amitriptyline inhibit nicotinic receptors in unmyelinated axons of human peripheral nerve. Br J Pharmacol 158:797-805

8. Frith J, Zalewski P, Klawe JJ, Pairman J, Bitner A, Tafil-Klawe M, Newton JL (2012) Impaired blood pressure variability in chronic fatigue syndrome-a potential biomarker. QJM 105:831-838
9. Hou RH, Langley RW, Szabadi E, Bradshaw CM (2007) Comparison of diphenhydramine and modafinil on arousal and autonomic functions in healthy volunteers. J Psychopharmacol 21:567-578

10. Khurana RK, Eisenberg L (2011) Orthostatic and non-orthostatic headache in postural tachycardia syndrome. Cephalalgia 31:409-415

11. Low PA, Benarroch EE (2008) Clinical autonomic disorders. Lippincott Williams \& Wilkins, Philadelphia

12. Lutgendorf SK, Latini JM, Rothrock N, Zimmerman MB, Kreder KJ Jr (2004) Autonomic response to stress in interstitial cystitis. J Urol 172:227-231

13. Mazur M, Furgala A, Jablonski K, Mach T, Thor P (2012) Autonomic nervous system activity in constipation-predominant irritable bowel syndrome patients. Med Sci Monit 18:CR493CR499

14. Mazurak N, Seredyuk N, Sauer H, Teufel M, Enck P (2012) Heart rate variability in the irritable bowel syndrome: a review of the literature. Neurogastroenterol Motil 24:206-216

15. Staud R (2008) Heart rate variability as a biomarker of fibromyalgia syndrome. Fut Rheumatol 3:475-483

16. Stav K, Lang E, Fanus Z, Leibovici D (2012) Autonomic response during bladder hydrodistention in patients with bladder pain syndrome. J Urol 188:117-121 